\title{
The Exploration of Financial Sharing Model for Large Groups-Based on the Perspective of Swot Analysis
}

\author{
Dan Han, Xuemei Chen* \\ School of International Business, Shanxi Normal University \\ Xi'an city, China
}

\begin{abstract}
Because of the economic globalization and China's "Belt and Road" initiative, large enterprise groups that willing to complete their transformation and improve their international competitiveness should construct a financial sharing model undoubtedly. The meanings of financial sharing service, the necessity of its establishing and construction strategy are the topics that scholars are now focusing on. By the means of Swot analysis, this paper analyzes the internal strengths, weaknesses, external opportunities and threats factors against the background of large groups building financial sharing model. Meanwhile, it also puts forward that large groups should fully grasp the opportunity and applies the advanced technology. Moreover, if the large groups have their own clear positioning and innovative services, and can overcome its own weaknesses and eliminate external threats, they could eventually exert the largest effects of financial sharing model.
\end{abstract}

Keywords-Financial sharing; Large groups; Swot analysis

\section{INTRODUCTION}

In recent years, with the globalization of the economy, the rapid development of science and technology and the convergence of accounting information, the conditions for enterprises to build a financial sharing model have continued to mature, and the internal and external environments have improved. More and more Chinese companies have turned their attention to finances sharing. However, before the establishment of the financial sharing model, we can use a strategic vision to examine the internal and external conditions for the implementation of this initiative. In the current new era, strategic thinking is necessary, which can be used to guide companies to implement and construct financial sharing models that meet their own characteristics under optimal conditions. Large group companies need to consider the major issues first, and swot analysis is one such method that can help companies use strategic analysis methods to make appropriate decisions. We hope that before making a decision to build a financial sharing center, companies should make decisions based on comprehensive consideration of their own capabilities and external environment so that they can better play their own advantages, avoid disadvantages, and seize external opportunities. The premise of risk avoidance is to build and implement a financial sharing model with its own characteristics.

\section{SWOT ANALYSIS}

\section{A. Strengths}

\section{1) Scale effect}

With the expansion of large-scale groups and the continuous increase of branch offices, the financial organizations of headquarters and branch offices are often set up repeatedly in that causing bloated organizations. This also increases the difficulty of managing the headquarters and the cost of the financial management work, and reduces the increase in the economic benefits brought by the scale expansion. Bryan Bergeron [1] (2003) believes that the main goal of financial sharing is to pursue the reduction and savings of various costs of the company. Li Yanyan believes that financial sharing services can achieve economies of scale and reduce business costs by centralizing and processing a large number of repetitive and simple businesses. Therefore, for a large group, if it constructs a financial sharing model, although the initial investment is more, the effect brought by the later period can actually increase the scale effect, greatly reduce the labor cost, and eliminate the specification of the business process. After repeated operations, the effect of reducing operating costs is even more pronounced in large groups.

\section{2) Internal synergies}

There are many departments and branches in large-scale groups, and the departments are closely related to each other. But there are differences in the actual situation in different places. There are no unified financial systems and processes in each branch office so they cannot cooperate well with each other, which will result in information being unable to provide timely feedback. Fahy [2] (2005) believes that financial sharing services can reduce unnecessary business processes by reengineering and standardizing business processes. At present, the sharing of external data between companies and partners has directly brought a lot of business innovations, such as the change of traditional models such as supply chain finance and bank-enterprise cooperation.

After streamlining the business process of the company, the relationship between the headquarters and the divisions can be more closely and orderly. It can make communication between the finance department and other functional departments more 
effective, which can also create a synergy effect within the large-scale group and further enlarge the effect of this effect.

\section{B. Weaknesses}

Zhang Ying [3] (2003) once mentioned the impact of shared services on Chinese enterprises. She thinks it is an opportunity as well as a challenge because the construction of the shared model will inevitably break the original organizational structure of the enterprise and even the original culture of the enterprise.

\section{1) Obstacles faced by the group employees}

Firstly, large-scale groups are usually formed through mergers and acquisitions. Because the differences of each company's corporate culture, it is difficult to form a unified culture, which are not conducive for the group to build a financial sharing service model. Many group employees simply believe that building a financial sharing model has no practical interest in themselves. Therefore, there is a lack of enthusiasm for building a financial sharing model or a weak awareness of shared services.

Furthermore, the construction of the financial sharing model will inevitably require employees to change their original work habits and learn new ways of working. Employees may not be willing to change themselves. Meanwhile, employees within the company who only care about their own interests may not be prepared for the relevant ideology or strongly oppose it.

\section{2) Obstacles in the implementation process}

The financial sharing model is an important way to improve the overall management quality and even economic benefits of the group. However, it is not able to make progress in the process of formulation. First, the management personnel of the financial sharing service center is not clear. Secondly, the workflow of each financial sharing mode is not standardized, which is not convenient for unified management. Finally, there is no evaluation link in the financial sharing model, but only through this link can we analyze and optimize the imperfect management system of the financial sharing model.

In addition, during the expansion of large-scale conglomerates, with the increasing scale and increasing number of branches, their internal problems are also increasing day by day. In particular, the formulation of management systems has become difficult to adjust, and it is difficult to establish uniform standards and norms. As a result, all branches and sub-branches have their own affairs, and the construction of a financial sharing model on this matter will inevitably lead to further increase in the difficulty of the management of the following organizations.

What is more, the construction of a financial sharing model is not an overnight event. It is difficult to reach a consensus during the implementation process, and it is prone to problems and frictions. It also takes some time to adapt and run in the implementation process. These uncertain factors have increased financial and operational risks. Second, if we do not pay attention to the combination of the current actual environment and blindly carry out the construction of a financial sharing model, we will also increase the risk that companies bear. Therefore, in the process of constructing a financial sharing model, large-scale groups may undertake a new process reengineering under a new technology background and may assume higher risk of failure.

\section{3) Obstacles in the information technology}

With the revolution of information technology, many more advanced information algorithms such as big data, cloud computing and artificial intelligence are born, which is extremely beneficial for the group to build a financial sharing center. Cheng Ping [4] (2016) and others provided directions and ideas on how to better construct and apply financial sharing models in the context of big data and cloud computing information technology. However, if the group wants to continuously optimize and apply these constantly updated information technologies, it will require a lot of costs and even more specialized talents, all of which require a lot of money and time. The investment is not conducive to the short-term development of the Group. Many groups only pay attention to immediate short-term interests, and do not consider the development of the group's financial management from a longterm perspective. As a result, they have only started to construct a financial sharing model, but they have not really studied and provided optimization support. Therefore, how to effectively implement the effective integration of corporate financial sharing models and emerging information technologies? How to standardize, universalize, and share financial sharing platforms and service models? How to deeply apply information technologies, and constantly optimize financial sharing service platforms and other issues? All these questions above need to be solved In-depth research and discussion.

\section{Opportunities}

\section{1) Good financial management environment}

In the context of the new era, the process of economic globalization has been further accelerated, and information technology has developed rapidly. All these provide a good environment for the group companies to open up markets and build financial sharing models. The continuous breakthrough of information technology makes the financial management work break the time and space constraints of information transmission. The company can achieve centralized accounting and synchronization feedback through the financial sharing platform. The financial resources are effectively integrated. Through the financial sharing model, the company can achieve Remote reimbursements, audits, and remote fund redeployment based on real-world needs, and the entire process can be monitored so that the enterprise accounting system and the enterprise's operating system seamlessly converge. E-currency has gradually become the main means of corporate transactions and payments. Under the management of financial sharing model, enterprises and supply chains can also be efficiently connected. Online inquiry, transaction and settlement can help enterprises further realize business finance. Convergence improves the overall operational efficiency and reduces costs. 


\section{2) Strong support of government}

In recent years, the Ministry of Finance has repeatedly issued relevant documents to advocate financial staff to accelerate the change of their own functions. Large enterprises and enterprise groups should fully grasp the favorable environment for economic growth and information technology development and actively build a financial sharing platform. At present, over $80 \%$ of Fortune 100 companies have built a financial sharing model. Many enterprises and groups in China, such as China Mobile, Haier and ZTE, have also achieved good results in applying the financial sharing model. However, it is necessary to establish and how to build a financial sharing service model. Each enterprise group must analyze and discuss after actual analysis and discussion.

\section{Threats}

\section{1) Lack of theoretical support}

Western developed countries have developed a rich theoretical system concerning financial sharing through diverse research methods such as literature research, case studies, empirical research, and field research. These include the basic concepts, concepts, advantages, and key elements of financial sharing. Practice. Domestic studies on the theory of financial sharing services started relatively late with respect to Western developed countries. There are few research results under the actual national conditions of our country, and it is easy to cause many companies to create deviations due to lack of theoretical guidance in the process of constructing financial sharing service models. Scholars at home and abroad pay more attention to the concrete practice of financial sharing, and both the theory and practice are mutual promotion and coordinated development. With the increasing construction and application of financial sharing in China, in recent years, Chinese scholars have made a lot of progress in the study of financial sharing, but most of them are qualitative studies or case studies, and their content is not comprehensive. The content and depth of research still far from enough, and the lack of support for related theories will naturally limit the pace of our practice.

\section{2) The imperfect internal control system}

Information security: Under the financial sharing model, the management of business and financial data is basically through information technology. If there are loopholes in the financial sharing platform or the corresponding financial software, it is difficult to ensure the security of the company's internal financial information.

Increased risk of internal control: In the implementation of the financial sharing model, the transfer of information and even the deployment of funds between parent and subsidiary companies and sub-headquarters companies are all concentrated on the financial sharing platform, and as the size of the company continues to expand, the group's original internal The control system is often imperfect, can not well match the financial sharing model, and is prone to problems that are sparse in prevention. Since China introduced and developed the financial sharing model for more than 30 years, the internal monitoring environment of the enterprise is weak, and many companies have not yet realized it. The additional risks that existed after the establishment of the financial sharing model did not establish a sound supervisory and control system in advance, which would increase the company's internal control risk.

\section{RECOMMENDATIONS}

\section{A. Formulating a budget}

Before a large-scale group builds a financial sharing model, it must be clear that the purpose of using this model for financial management is to improve the level of financial management and reduce corporate management costs. If the use of financial sharing model management costs is too high, even more than the benefits it brings, it must be carefully considered whether it is necessary for the group to build a financial sharing model. This mainly takes into account the need to purchase financial software, introduce new information technologies, staff training, and hardware purchases to adopt the new financial sharing model. Therefore, the cost of implementing the financial sharing model is very high. Companies should consider their implementation costs before implementation. With the benefits and other aspects of the impact, avoiding gains and losses.

\section{B. Increasing participation and sharing awareness}

In the process of implementing the financial sharing model, the company, department, or other business unit may does not cooperate. Therefore, the enterprises must transformed their culture and management decision-making methods first, and adopt certain strategies to promote them accept the shared service model. First of all, it is necessary to increase publicity and training in the financial sharing model and make employees aware of the financial sharing model through multiple channels. For example, in the process of its construction, large-scale groups can include the relevant process positions and their personnel in the financial sharing center, enabling more. Employees can participate in it; the second is to deepen the financial communication and coordination of various departments, and lay a foundation for building a financial sharing model. Finally, management must learn more about the financial sharing model, and support the business department, information technology department, etc. All departments actively and effectively communicate with each other to facilitate the subsequent landing of the project. And the group should fully respect the personal wishes of employees, and encourage them to develop in the direction of the backbone of the business. In this way, the employees can only change the consciousness of all employees of large-scale groups.

The training of employees and teams is a continuous process. The transformation and promotion of employees is a long-term process. Excessive urgency often fails to achieve the desired effect. The group can make plans in advance and promote employees' participation in the construction of the financial sharing model step by step. 


\section{Training qualified financial managers}

With the rapid development of science and technology and the continuous impact on the finance and accounting industry, society and companies have placed higher demands on financial personnel. On the one hand, financial personnel need to learn and understand more computer network technologies. On the other hand, they also need to be familiar with the activities of the company's operations and operations. If financial personnel can better grasp information technology and data knowledge, and can independently analyze the underlying data to achieve a complete convergence of finance and information technology, it will greatly reduce the omission of key value information and provide a solid foundation for the construction of financial sharing model.

Enterprises must have enough patience and perseverance to regard the establishment of an effective personnel training mechanism as a long-term task, and to regard the cultivation of talents and the construction of a financial sharing model as a long-term strategic investment, and gradually and orderly. At the same time, the establishment of the financial sharing model is not only the work of the financial department, but also requires the active participation of all departments of the company, especially the recognition, understanding and strong support of the leaders at the highest level, to provide a good environment for financial personnel's transformation and to give them Professional Training.

\section{Standardizing the management system}

Perfecting the financial management system and formulating standardized processes are prerequisites for the construction of the financial sharing service model. First, at the group level, the financial management system was established at night, and standardized processes were established as the basis for building a financial sharing model. Secondly, through centralized training, all branches can keep abreast of the new standards and lay the foundation for formal implementation. Finally, continuous supervision and enforcement are the guarantee for the smooth implementation of the management system.

The establishment of an effective internal control system is the key to rapid financial sharing. Based on the financial sharing model established by the large-scale group itself, a corresponding internal control and risk warning mechanism is established to optimize the design of the internal control process. First of all, to ensure information security, the second is to focus on prevention of internal control risks. Large-scale groups must improve their own risk awareness and formulate preventive measures. After the financial sharing model is established, the information transmission between large-scale groups depends entirely on computer network technology. In the process of transferring information, we must guard against potential risks and build a sound supervision and control system so that effective supervision and timely feedback can be provided.

Reforming the financial process is a necessary means to achieve the integration of the business and data of the financial shared service center. The essence of financial sharing services is the sharing of processes, and their construction and implementation must follow the basic view of BPR theory (Lusk, 1999) [5]. Therefore, in the process of constructing a financial sharing model, continuous financial process improvement should be a major concern for large groups.

\section{E. Ensuring the matching of information technology}

The Group's construction of a financial sharing model is a change in the internal organizational structure and an upgrade of the existing financial management system. The integration of the financial management system and information technology can, on the one hand, improve the financial management of the group, and on the other hand provide a strong background support for group management and decision-making. Therefore, the effective operation of the financial sharing model is inseparable from the support of informationization. The supporting of information technology is the key guarantee for successfully building a financial sharing model. However, the emerging technologies such as big data and cloud computing in the new era are both a challenge and an opportunity for the financial sharing model.

Therefore, large-scale groups should proceed from their actual conditions, improve the information system construction supported by the financial sharing model, make full use of information technology to promote the development of financial sharing model, and effectively improve the group's management efficiency and value creation capabilities.

\section{CONCLUSION}

The establishment of a financial sharing model will be the trend of the financial management model for large-scale group reform in China. The financial sharing model is not only an organizational form of advanced information technology, but also an innovative and efficient management model. It can effectively reduce the company's operating costs and improve the group's overall benefits. It is essential for companies to increase their overall strength. At present, the financial sharing model has not been widely used in China. Only a few largescale groups have explored the construction of the financial sharing model to some extent. However, as China's corporate scale continues to expand and businesses continue to expand externally, the role of the financial sharing model as an important core competence has become increasingly prominent. With the strong support of national policies and the promotion of a new era, China will have more enterprise groups in the future and choose to build a financial sharing model to further enhance its core competitiveness and move toward a better posture. The center of the stage.

\section{ACKNOWLEDGEMENT}

This research was financially supported by research on the integration and innovation development mechanism of finance and cultural industry Project of National social science foundation youth program, China, Project Number: 12CJL032;Humanities and Social Sciences Research Plan Fund Project of Education Ministry, Project Number: 15YJA630052; Fundamental Research Funds of Central Universities of Shaanxi Normal University, Project Number: 10SZYB26. 


\section{REFERENCES}

[1] Bergeron B.2003.Essentials of knowledge management. Technical Communication.51 (3):425-426.

[2] Frawley T, Fahy J.2006.Revisiting the first-mover advantage theory: a resource-based perspective. Irish Journal of Management, 273-295.

[3] Ying Zhang. Gaofeng Zhang, Wei lv. 2003. New "background" service sharing center for enterprises. Enterprise reform and management, (2) 11-12. (In Chinese)

[4] Ping Cheng, Shan Chen. 2016. Monetary fund management of financial sharing service center based on cloud accounting. Friends of accounting, (14)129-132. (In Chinese)

[5] Luck, Schulman D S, Dunleavy J R, Harmer M J, et al. 1999.Shared Services: Adding Value to the Business Units. Jhon Wiley \& Sons. 